\title{
The Extra Differential Gain Enhancement in Multiple-Quantum-Well Lasers
}

\author{
B. Zhao, T. R. Chen, and A. Yariv
}

\begin{abstract}
By accounting for the unavoidable thermal population of injected carriers in the optical confining layers we find that the use of multiple quantum wells (MQW) as active region actually leads to an extra increase in differential gain. Specifcally, the maximum differential gain increases with the number of wells in the quantum-well structures. The transparency current density in the MQW structures does not scale as the number of quantum wells. These conclusions are at variance with presently accepted theory and of major implications for the design of high-speed, low-threshold semiconductor lasers.
\end{abstract}

$\mathrm{Q}$ UANTUM-well (QW) lasers have become the main contenders for high frequency $(>10 \mathrm{GHz}$ ) modulation This is due mostly to the predicted differential gain enhancement in the QW lasers compared to equivalent bulk devices [1]. However, high-speed modulation experiments to date show no improvement in the unstrained single quantum-well (SQW) structure lasers [2], [3]. In conventional QW laser structures, a separate confinement heterostructure ( $\mathrm{SCH})$ is used to obtain optical confinement of the optical field and electronic confinement of the injected carriers. A reexamination of the theory shows that the neglect of carrier population in the optical confining layers (CL) is not justified in the typical structures used in conventional QW lasers. An inclusion of the CL population in the analysis leads to computed differential gain in room temperature SQW lasers which is lower than that in their bulk counterparts [4]. This reduction is due to the finite (and appreciable) Fermi occupation factor for the states near the (energy) bottom of the CL and to the large density of such states compared to the QW. The expectation of improved dynamics of QW lasers is significantly affected by the carrier population of the higher energy states (state-filling) in the QW structures [5].

To date, it is well known that above transparency the differential gain decreases as the carrier density increases in QW structures because of the sublinearity in the gain versus carrier density dependence. This sublinearity is attributed to the flat feature of the two-dimensional step-like density of states in the QW structures. For a given value of modal gain, the differential gain increases as the number of quantum wells ( $N_{\text {qw }}$ ) increases because the carrier density associated with one single QW decreases with the increase of $N_{\mathrm{aw}}$. This is

Manuscript received October 8, 1991; revised December 2, 1991. This work was supported by DAPPA, the Office of Naval Research, and the Air Force Office of Scientific Research.

The authors are with the T. J. Watson Sr. Laboratories of Applied Physics, 128-95, California Institute of Technology, Pasadena, CA 91125.

IEEE Log Number 9105983. the conventional differential gain enhancement in MQW structures. Without inclusion of the state-filling effect, it has been predicted that larger differential gain should be achieved at lower carrier density (or at smaller modal gain) and that the maximum attainable differential gain is not affected by the number of quantum wells [6]. In this letter, we show that the inclusion of confining layer state-filling considerably modifies this picture. Our analysis predicts that the use of multiple quantum wells (MQW) as active region actually leads to an extra differential gain enhancement which has not been noticed before. More specifically, the maximum attainable differential gain increases as the number of quantum wells increases. These results agree with experimental observations of differential gain enhancement in MQW structures [7]-[9] and improved high frequency performance over SQW in MQW lasers [3], [10]-[12].

The physical reason for the extra increase in differential gain in MQW structures is the following. In a SQW laser the need to obtain a sufficient optical gain (to overcome the losses) forces the Fermi energy $E_{f}$ to rise toward the top of the quantum well as the pumping level is increased. The confining layer states, say at energy $E_{c l}$, whose occupation is determined by the factor $\exp \left[-\left(E_{c l}-E_{f}\right) / k T\right]$ are thus more heavily populated. This increase in the $\mathrm{CL}$ carrier density contributes negligibly to the increase in gain because of the large nonresonant nature of these transitions. This leads to reduced differential gain. In a MQW laser, on the other hand, the necessary total gain is reached with a much lower population in any one well. This results in a much lower $E_{f}$ and consequently the $\mathrm{CL}$ states occupation factor $\exp \left[-\left(E_{c i}-E_{f}\right) / k T\right]$ is smaller. If we represent the CL states by a fixed number $D$ (equal to the total number of states per unit area within $k T$ from the top of the quantum wells) and take the bottom of the quantum well $n=1$ state energy as $E=0$, it is a straightforward task to show that the differential gain is given by [5]

$$
\begin{aligned}
\frac{d G_{0}}{d c_{e}}= & \frac{A_{0}}{k T} \frac{1}{1+\exp \left(\frac{E_{f}}{k T}\right)} \\
& \cdot \frac{1}{1+\frac{D}{N_{\mathrm{qw}}} \frac{1+\exp \left(-E_{f} / k T\right)}{1+\exp \left[\left(E_{c l}-E_{f}\right) / k T\right]}}
\end{aligned}
$$

where $G_{0}$ is the two-dimensional (2-D) optical gain, $A_{0}$ is a material-dependent parameter and $c_{e}$ is the 2-D carrier density. Note that the increase in $d G_{0} / d c_{e}$ with increasing 
number of wells $\left(N_{\mathrm{qw}}\right)$ is due to the explicit dependence on $N_{\mathrm{qw}}$ as well as to the implicit dependence on $E_{f}$ since the latter decreases with increasing $N_{\mathrm{qw}}$. The explicit term $D / N_{\text {qw }}$ in (1) shows that the penalty due to CL state-filling is reduced by "sharing"' it with $N_{\mathrm{qw}}$ quantum wells in MQW structures.

The model leading to (1) contains all the relevant physics but is simplistic. To get more detailed and accurate results, we carried out a gain calculation assuming a typical unstrained GaAs/AlGaAs SCH QW laser structure, which accounts for the actual population distribution in the $\mathrm{QW}$ and CL regions. This analysis applies as well to other material systems such as strained QW lasers. The linear 2-D optical gain at a photon energy $E$ under a $k$-selection rule can be taken as

$$
\begin{aligned}
G_{0}(E)= & N_{\mathrm{qw}} B \frac{E}{n_{r}} \sum_{i} \int \delta_{i} \rho_{i, r}^{\mathrm{SQw}}(\epsilon)\left(f_{e}+f_{h}-1\right) \\
& \cdot \frac{\hbar / T_{2}}{\left(\hbar / T_{2}\right)^{2}+(\epsilon-E)^{2}} d \epsilon+B \frac{E}{n_{r}} \\
& \cdot \sum_{i} \int \delta_{i} \rho_{i, r}^{\mathrm{cL}}(\epsilon)\left(f_{e}+f_{h}-1\right) \\
& \cdot \frac{\hbar / T_{2}}{\left(\hbar / T_{2}\right)^{2}+(\epsilon-E)^{2}} d \epsilon
\end{aligned}
$$

where $T_{2}$ is the collisional broadening time, $f_{e}$ and $f_{h}$ are the quasi-Fermi distribution functions for electrons and for holes respectively, $\rho_{i, r}^{\mathrm{SQW}}$ is the step-like 2-D reduced density of states for the carriers in one single $\mathrm{QW}, N_{\mathrm{qw}}$ is the number of the quantum wells as defined above, we have assumed that the quantum wells are identical and uncoupled, $\rho_{i, r}^{\mathrm{CL}}$ is the reduced density of states for the carriers in the confining layer region, $i$ designates the corresponding subbands, $\delta_{i}$ is the polarization factor for the dipole moment in the QW structure [13], $n_{r}$ is the modal effective refractive index, and $B$ is a constant.

The carrier density and quasi-Fermi functions are related by quasi-neutrality $c_{e}=c_{h} . c_{e}$ and $c_{h}$ are the total 2-D electron density and hole density, respectively, in the SCH QW structure

$$
\begin{aligned}
c_{e}=N_{\mathrm{qw}} \sum_{i} \int \rho_{i, e}^{\mathrm{SQw}}(\epsilon) f_{e}(\epsilon) d \epsilon & \\
& +\sum_{i} \int \rho_{i, e}^{\mathrm{CL}}(\epsilon) f_{e}(\epsilon) d \epsilon \\
c_{h}=N_{\mathrm{qw}} \sum_{i} \int \rho_{i, h}^{\mathrm{SQW}}(\epsilon) f_{h}(\epsilon) d \epsilon & \\
& +\sum_{i} \int \rho_{i, h}^{\mathrm{CL}}(\epsilon) f_{h}(\epsilon) d \epsilon .
\end{aligned}
$$

The typical SCH QW structure parameters used in our model correspond to a symmetric $4000 \AA$ $\mathrm{Al}_{0.5} \mathrm{Ga}_{0.5} \mathrm{As} / \mathrm{Al}_{0.2} \mathrm{Ga}_{0.8} \mathrm{As}$ graded index separate confinement heterostructure (GRIN-SCH) with $100 \AA$ GaAs quantum wells located at its center. The differential gain $G_{0}^{\prime}=$ $d G_{0} / d c_{e}$ is calculated at the peak of $G_{0}(E)$ for the dominant

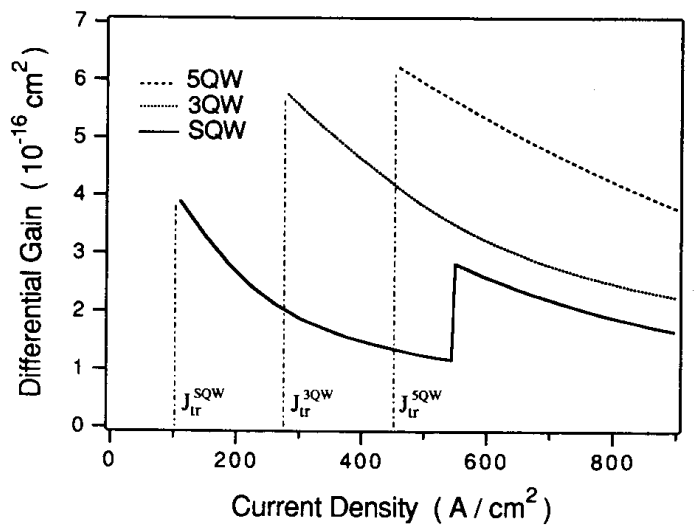

Fig. 1. The differential gain $d G_{0} / d c_{e}$ at gain peak above transparency as a function of injection current density in the QW structures with different number of quantum wells. $J_{t r}^{\mathrm{SQW}}, J_{t r}^{3 \mathrm{QW}}$ and $J_{t r}^{\mathrm{SQW}}$ are the corresponding transparency current densities in the different $\mathrm{QW}$ structures.

TE mode of this structure. We have assumed parabolic and uncoupled subbands. Typical values of effective masses for electrons and holes in GaAs material are employed and $T_{2}=0.1 \mathrm{ps}$ is used. In Fig. 1 we show the differential gain at the gain peak as a function of injected current density above transparency. The differential gain is calculated for GRINSCH QW laser structures with a single quantum well (SQW), three quantum wells ( $3 \mathrm{QW}$ ) and five quantum wells $(5 \mathrm{QW})$, respectively. A carrier lifetime of $4 \mathrm{~ns}$ is used in the evaluation of the current density. Fig. 1 shows clearly that the maximum attainable differential gain above transparency increases as the quantum-well number increases.

The results in Fig. 1 are different from the currently accepted picture concerning the influence of quantum-well number on the differential gain as we mentioned above [6]. For the sake of comparison we show in Fig. 2 a plot of the differential gain which results if we neglect the "sharing" of CL state filling among the $N_{\mathrm{qw}}$ quantum wells. Note that the maximum differential gain above transparency is independent of the number of quantum wells.

Fig. 1 shows how the increase in $N_{\mathrm{qw}}$ can result in a larger differential gain. Since the current density in the laser is determined by the gain needed to overcome the total optical resonator losses it is useful to recast the information of Fig. 1 using the modal gain as the abscissa. To do that we have evaluated the transverse effective optical mode width as $0.28 \mu \mathrm{m}$ for the assumed GRINSCH structure. The modal gain is obtained by the 2-D gain constant $G_{0}$ divided by the effective optical mode width of the GRINSCH structure. The results are shown in Fig. 3. Note that a $N_{\mathrm{qw}}=5$ laser with a loss of $40 \mathrm{~cm}^{-1}$, for example, will have a differential gain which is appreciably larger than the maximum attainable differential gain in a SQW laser and nearly three times as large as a SQW laser with the same loss value.

These results are in good agreement with the experimental observations in QW laser structures. In experiments, it is not easy to compare the differential gain among QW structures with different number of quantum wells since the differential 


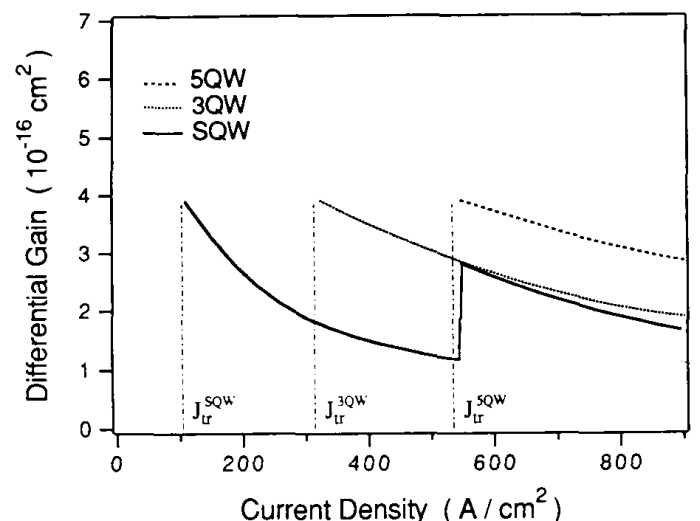

Fig. 2. The differential gain $d G_{0} / d c_{e}$ at gain peak as a function of injection current density in the QW structures with different number of quantum wells. The gain is computed incorrectly, see the context.

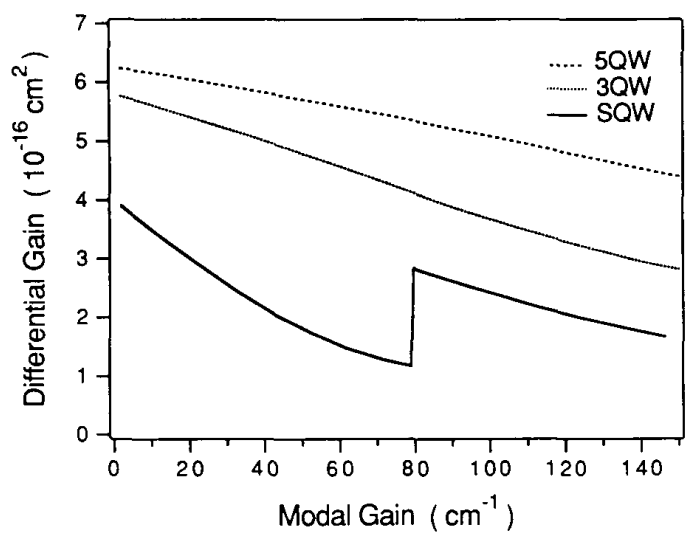

Fig. 3. The differential gain $d G_{0} / d c_{e}$ at gain peak as a function of modal gain in the $\mathrm{QW}$ structures with different number of quantum wells.

gain is strongly dependent on the injected carrier density (or the threshold modal gain). Recently, however, it is found experimentally that there is a definite trend of increasing differential gain with increasing the number of quantum wells [14]. In addition to the relative fading of the damping effect and the better operation conditions to achieve the maximum modulation speed in MQW structures [15], this extra differential gain enhancement is also responsible for the improved high speed performance in MQW lasers.

Finally, we point out that the transparency current density $\left(J_{\mathrm{tr}}\right)$ in MQW structures is not scaled as $J_{\mathrm{tr}}^{\mathrm{MQW}}=N_{\mathrm{qw}} J_{\mathrm{tr}}^{\mathrm{SQW}}$. As shown in Fig. 1, the penalty of using MQW as active region on $J_{\mathrm{tr}}$ is smaller than that scaling. This results from the fact that the carrier population in the $\mathrm{CL}$ region is not scaled by $N_{\mathrm{qw}}$ in the MQW structures at transparency.
In conclusion, it is shown that the use of MQW structure as active region in $\mathrm{SCH}$ QW laser structures leads to an extra differential gain enhancement. This extra differential gain enhancement results from the reduction of the state-filling effects in the confining layers. These results are consistent with the experimentally observed trend of increasing differential gain with increasing quantum-well number and the improved high-speed performance in MQW lasers. We find that the transparency current density in MQW lasers does not scale as the number of quantum wells. The penalty on transparency current density $J_{\mathrm{tr}}$ due to the use of MQW as active region is smaller.

\section{REFERENCES}

[1] Y. Arakawa and A. Yariv, "Quantum well lasers-gain, spectra, dynamics," IEEE J. Quantum Electron., vol. QE-22, pp. $1887-1899,1986$

[2] K. Y. Lau, N. Bar-Chaim, I. Ury, and A. Yariv, "11-GHz direct modulation bandwidth GaAlAs window laser on semi-insulating substrate operating at room temperature," Appl. Phys. Lett., vol. 45. pp. $316-318,1984$

[3] P. L. Derry, T. R. Chen, Y. H. Zhuang, J. Paslaski, M. Mittelstein, K. Vahala, and A. Yariv, "Spectral and dynamic characteristics of buried-heterostructure single quantum well (Al,Ga)As lasers," $\mathrm{Appl}$. Phys. Lett., vol. 53, pp. 271-273, 1988.

[4] B. Zhao, T. R. Chen, and A. Yariv, "Comparison of differential gain in single quantum well and bulk double heterostructure lasers," to be published.

[5] - "The effect of state-filling on the modulation response and the threshold current of quantum well lasers," to be published.

[6] Y. Arakawa and A. Yariv, "Theory of gain, modulation response, and spectral linewidth in AlGaAs quantum well lasers," IEEE $J$. Quantum Electron., vol. QE-21, pp. 1666-1674, 1985.

[7] K. Uomi, N. Chinone, T. Ohtoshi, and T. Kajimura, "High relaxation oscillation frequency (beyond $10 \mathrm{GHz}$ ) of GaAlAs Multiquantum well lasers." Japan. J. Appl. Phys., vol. 24, pp. L539-L541, 1985.

[8] K. Uomi, T. Mishima, and N. Chinone, "Ultrahigh relaxation oscillation frequency (up to $30 \mathrm{GHz}$ ) of highly p-doped $\mathrm{GaAs} / \mathrm{GaAlAs}$ multiple quantum well lasers," Appl. Phys. Lett., vol. 51, pp. $78-80,1987$

[9] T. Takahashi, M. Nishioka, and Y. Arakawa, “Differential gain of GaAs/AIGaAs quantum well and modulation-doped quantum well lasers," Appl. Phys. Lett., vol. 58, pp. 4-6, 1991

[10] H. D. Wolf, H. Lang, and L. Korte, "High-speed AlGaAs/GaAs multiple quantum well ridge waveguide lasers," Electron. Lett., vol. 25, pp. 1245-1246, 1989.

(11] T. R. Chen, L. E. Eng, B. Zhao, Y. H. Zhuang, S. Sanders, H. Morkoç, and A. Yariv, "Submilliamp threshold InGaAs-GaAs strained layer quantum-well laser," IEEE J. Quantum Electron., vol. QE-26, pp. $1183-1190,1990$

[12] R. Nagarajan, T. Fukushima, J. E. Bowers, R. S. Geels, and L. A. Coldren, "High-speed InGaAs/GaAs strained multiple quantum well lasers with low damping," Appl. Phys. Lett., vol. 58, pp. 2326-2328, 1991.

[13] M. Yamada, S. Ogita, M. Yamagishi, K. Tabata, N. Nakaya, M. Asada, and Y. Suematsu, "Polarization-dependent gain in GaAs/AlGaAs multi-quantum-well lasers: Theory and experiment," Appl. Phys. Lett., vol. 45, pp. 324-325, 1984.

[14] M. C. Tatham, C. P. Seltzer, S. D. Perrin, and D. M. Cooper, "Frequency response and differential gain in strained and unstrained InGaAs/InGaAsP quantum well lasers," Electron. Lett., vol. 27, pp. $1278-1280,1991$

[15] B. Zhao, T. R. Chen, and A. Yariv, "On the high speed modulation bandwidth of quantum well lasers," Appl. Phys. Lett., vol. 60, 1992 